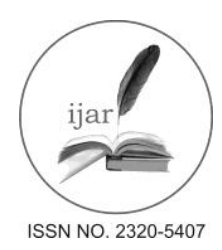

Journal homepage:http://www.journalijar.com
Journal DOI:10.21474/IJAR01

RESEARCH ARTICLE
INTERNATIONAL JOURNAL

OF ADVANCED RESEARCH

מחיח

\title{
A CASE REPORT : TETANUS AFTER PILES SURGERY BY A QUACK DOCTOR IN PERIPHERAL MAHARASHTRA.
}

\author{
V.S. Rathod ${ }^{1}$, Rohit Sinha ${ }^{2}$, ${ }^{*}$ Shuvankar Mukhopadhyay ${ }^{3}$, Kasturi $^{2}$, Tamboli S ${ }^{1}$, V Shegokar ${ }^{4}$. \\ 1. Associate Professor, Microbiology Department, Dr SC Gov Medical College, Nanded,Maharashtra. \\ 2. Resident,Microbiology Department, Dr SC Gov Medical College, Nanded,Maharashtra. \\ 3. Assistant Professor,Microbiology Department, Dr SC Gov Medical College, Nanded,Maharashtra. \\ 4. Professor\& HOD, Microbiology Department, Dr SC Gov Medical College, Nanded,Maharashtra
}

\section{Manuscript Info \\ Manuscript History: \\ Received:14 April 2016 \\ Final Accepted: 19 May 2016 \\ Published Online: June 2016 \\ Key words: \\ Tetanus ,piles ,surgery ,quack. \\ *Corresponding Author}

\begin{abstract}
Postsurgical tetanus was a common phenomenon before era of tetanus prophylaxis .But still it is a problem of developing country like India specially in rural area where many people neglect vaccination programme and where surgery is done without any aseptic precaution by village quack. Here we report a case of Tetanus acquiring during piles surgery by a untrained quack doctor in peripheral Maharashtra.
\end{abstract}

\section{Introduction:-}

Tetanus was well known to ancient people who recognized the relationship between wounds and fatal muscle spasms. ${ }^{1}$ In 1884, Arthur Nicolaier isolated the strychnine-like toxin of tetanus from free-living, anaerobic soil bacteria. The etiology of the disease was further elucidated in 1884 by Antonio Carle and Giorgio Rattone, two pathologists of the University of Turin, who demonstrated the transmissibility of tetanus for the first time. They produced tetanus in rabbits by injecting pus from a patient with fatal tetanus into their sciatic nerves. ${ }^{2}$

In 1891, C. tetani was isolated from a human victim by KitasatoShibasaburō, who later showed that the organism could produce disease when injected into animals, and that the toxin could be neutralized by specific antibodies. ${ }^{3}$

Tetanus is caused by an infection with the bacteriumClostridium tetani, ${ }^{[1]}$ It is also known as lockjaw, an infection characterized by muscle spasms. In the most common type, the spasms begin in the jaw and then progress to the rest of the body.

Postsurgical tetanus was a common phenomenon before era of tetanus prophylaxis .But still it is a problem of developing country like India specially in rural area where some people neglect vaccination programme and where surgery is done without any aseptic precaution by village quack. Here we report a case of Tetanus acquiring during piles surgery by a untrained quack doctor in peripheral Maharashtra

\section{Case presentation:-}

A 45 years old male patient waspresented with advanced stage of respiratory failure with lock jaw in tertiary care hospital in Peripheral Maharashtra.Inspite of best effort from medical team the patient died due to respiratory failure. Relatives of the patient informed that the patient was suffering from prolapsed piles for last 1 year. He was treated by ointment by local quack and 10 days before he was undergone surgery by the same quack. After eight 
days of surgery the patient suffered problem in opening the jaw and tonic contraction of the body, difficulty in daefecation, urination and breathing. Patient was taken to quack doctor where he was given some medicines. When respiratory troublesincreasedrelative thought that now it was the time to go to hospital. He was taken to our hospital in very advanced stage of respiratory failure and in spite of mechanical ventilation and anti tetanus immunoglobulin patient died on same day. Next day during autopsy perianal tissue biopsy was taken and send to microbiology department We performed gram staining of smear but no spore bearing organism was seen except some gram negative organism and pus cell. The tissue was black in color and was inoculated in Robertson cooked meat media in anaerobic condition.After7 days of inoculation the Robertson cooked meat media turned black. On gram staining of smear made from Robertson broth showed spore bearing organism having round terminal spore typical of Clostridium tetani(Fig1) A police case was filed against the quack doctor who treated the patient.

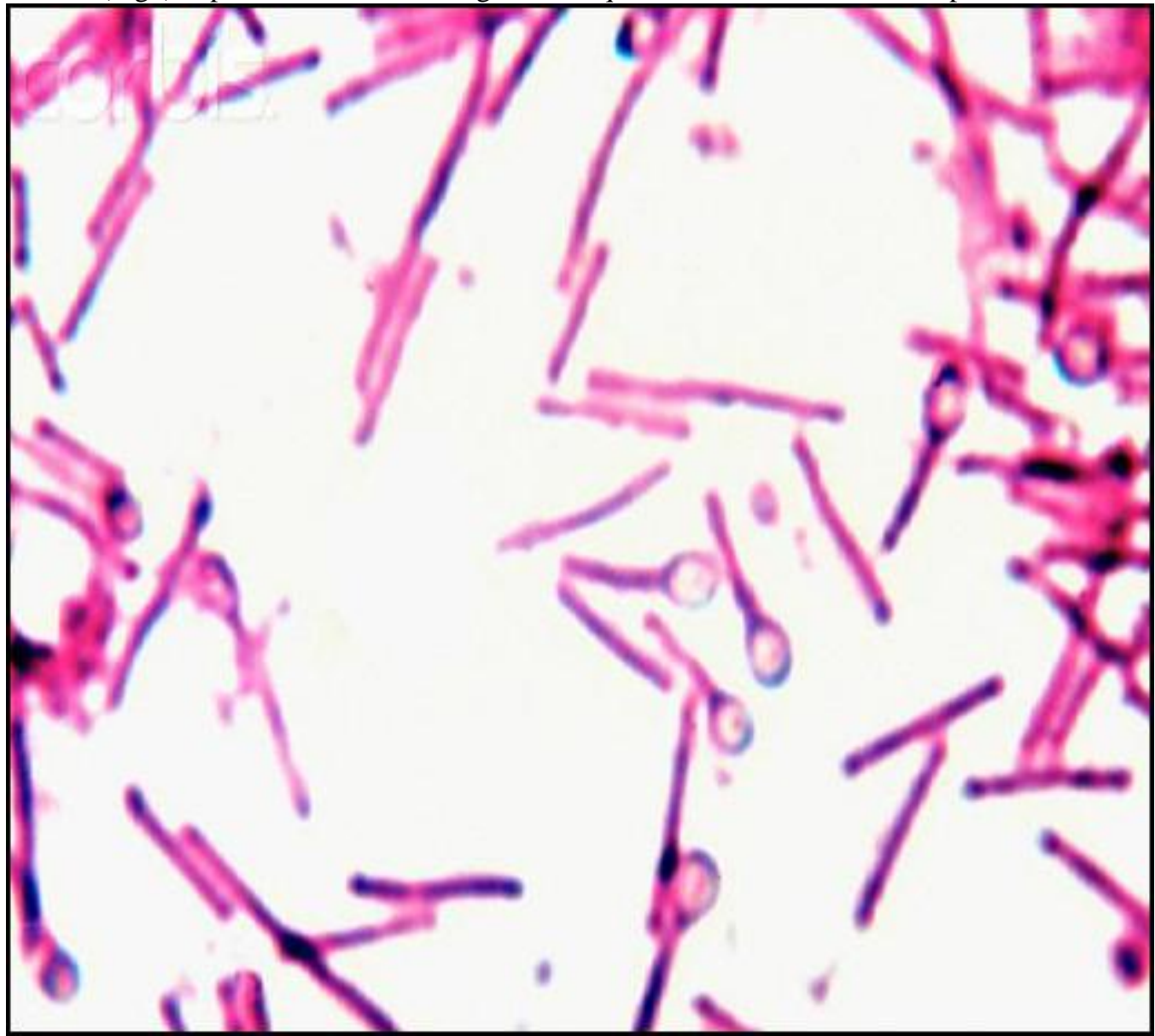

Figure 1:- Gram stain of smear made from RCM after 7 Days(100× MAGNIFICATION)

\section{Discussion:-}

Tetanus is an international health problem, as $C$. tetani spores are ubiquitous. The disease occurs almost exclusively in persons unvaccinated or inadequately immunized ${ }^{3}$ It is more common in hot, damp climates with soil rich in organic matter. This is particularly true with manure-treated soils, as the spores are widely distributed in the intestines and feces of many animals such as horses, sheep, cattle, dogs, cats, rats, guinea pigs, and chicken ${ }^{2}$ Spores can be introduced into the body through puncture wounds. In agricultural areas, a significant number of human adults may harbor the organism ${ }^{2}$

Tetanus affects skeletal muscle, a type of striated muscle used in voluntary movement. The other type of striated muscle, cardiac, or heart muscle, cannot be tetanized because of its intrinsic electrical properties.

The tetanus toxin initially binds to peripheral nerve terminals. It is transported within the axon and across synaptic junctions until it reaches the central nervous system. There it becomes rapidly fixed to gangliosides at the presynaptic inhibitory motor nerve endings, and is taken up into the axon by endocytosis. The effect of the toxin is 
to block the release of inhibitory neurotransmittersglycine and gamma-aminobutyric acid (GABA) across the synaptic cleft, which is required to check the nervous impulse. If nervous impulses cannot be checked by normal inhibitory mechanisms, the generalized muscular spasms characteristic of tetanus are produced. The toxin appears to act by selective cleavage of a protein component of synaptic vesicles, synaptobrevin II, and this prevents the release of neurotransmitters by the cell ${ }^{4}$

Post surgical tetanus was not uncommon before era of immunization and modern surgical practice with proper antiseptic precaution Now it is becoming rarest event. Only sporadic events of post surgical tetanus have been reported from peripheral rural area in developing country where still patient depends on quack doctor for treatment . Here in our case the patient was undergone piles operation by a quack doctor without under any aseptic precaution and the patient was also not immunized. After 7 days of operation (which denotes the incubation period of Clostridium tetani) the patient developed lock jaw and muscular contraction which were another features of tetanus but was not taken to hospital.He came to our hospital in very last stage and died due to respiratory failure.

This case clearly indicates that still in this era of modern medicine some people specially in rural area are not aware of importance of immunization and also they still depend on local quack doctor for treatment. We should organize some campaigns in rural area of developing world to increase awareness in public regarding this issue.

\section{References:-}

1. Pearce JM (1996). "Notes on tetanus (lockjaw)". Journal of Neurology, Neurosurgery, and Psychiatry 60 (3): 332. doi:10.1136/jnnp.60.3.332. PMC 1073859. PMID 8609513

2. "Tetanus" (PDF). CDC Pink Book. Retrieved 2007-01-26

3. Atkinson, William (May 2012). Tetanus Epidemiology and Prevention of Vaccine-Preventable Diseases (12 ed.). Public Health Foundation. pp. 291-300

4. Wells, C. L., Wilkins, T. D. (1996). "Clostridia: Sporeforming Anaerobic Bacilli". In Baron, S.; et al. Baron's Medical Microbiology. Univ of Texas Medical Branch. ISBN 0-9631172-1-1. 